\title{
ApoA-I mutations, L202P and K131del, in HDL from heterozygotes with low HDL-C
}

Stefan Ljunggren, Johannes H M Levels, Maria V Turkina, Sofie Sundberg, Andrea E

Bochem, Kees Hovingh, Adriaan G Holleboom, Mats Lindahl, Jan Albert Kuivenhoven and Helen Karlsson

\section{Linköping University Post Print}

\section{Tweet}

N.B.: When citing this work, cite the original article.

Original Publication:

Stefan Ljunggren, Johannes H M Levels, Maria V Turkina, Sofie Sundberg, Andrea E Bochem, Kees Hovingh, Adriaan G Holleboom, Mats Lindahl, Jan Albert Kuivenhoven and Helen Karlsson, ApoA-I mutations, L202P and K131del, in HDL from heterozygotes with low HDLC, 2014, PROTEOMICS - Clinical Applications, (8), 3-4, 241-250.

http://dx.doi.org/10.1002/prca.201300014

Copyright: Wiley-VCH Verlag http://www.wiley-vch.de/publish/en/

Postprint available at: Linköping University Electronic Press http://urn.kb.se/resolve?urn=urn:nbn:se:liu:diva-105992 


\section{APOA-I MUTATIONS, L202P and K131DEL, IN HDL FROM HETEROZYGOTES WITH LOW HDL- CHOLESTEROL}

Stefan Ljunggren ${ }^{1}$ Johannes H.M. Levels ${ }^{2}$ Maria V. Turkina ${ }^{3}$ Sofie Sundberg $^{1}$ Andrea E. Bochem ${ }^{2}$, Kees Hovingh ${ }^{2}$ Adriaan G. Holleboom ${ }^{2}$ Mats Lindahl ${ }^{1}$ Jan $^{2}$ Albert Kuivenhoven ${ }^{4}$ and Helen Karlsson ${ }^{1,5}$ \&.

${ }^{1}$ Occupational and Environmental Medicine, Department of Clinical and Experimental Medicine, Linköping University, Linköping, Sweden, ${ }^{2}$ Department of Vascular Medicine, Academic Medical Centre, Amsterdam, The Netherlands, ${ }^{3}$ Cell Biology, Department of Clinical and Experimental Medicine, Linköping University, Linköping, Sweden, ${ }^{4}$ University of Groningen, University Medical Center Groningen, Molecular Genetics, Groningen, The Netherlands, ${ }^{5}$ Occupational and Environmental Medicine, Heart Medical Centre, County Council of Östergötland, Linköping, Sweden §Corresponding author

Email addresses:

helen.m.karlsson@liu.se $\quad$ stefan.ljunggren@liu.se

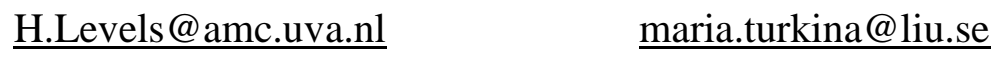

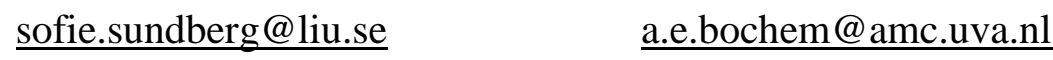

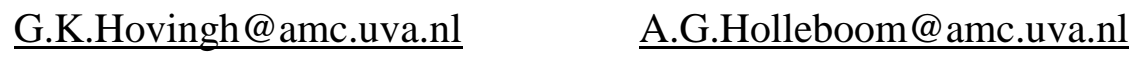

mats.lindahl@liu.se $\quad$ j.a.kuivenhoven@umcg.nl

Abbreviations: ABCA1, ATP-binding cassette A1, Apo, apolipoprotein, CVD, Cardiovascular disease, HDL, high-density lipoprotein, HDL-C, high-density lipoprotein cholesterol, LDL, low density lipoprotein, SRB1, scavenger receptor B-1, VLDL, very low-density lipoprotein

Keywords: Apolipoprotein A-I, High-density lipoprotein, apoA-I ${ }^{\mathrm{K} 131 \mathrm{del}}$, apoA-I ${ }^{\mathrm{L} 202 \mathrm{P}}$, Two-dimensional gel electrophoresis

Total number of words: 6140 


\section{Statement of clinical relevance}

Cardiovascular disease (CVD) has become the major cause of death worldwide. Atherosclerosis, the underlying disease ultimately leading to CVD events, is influenced by life style factors such as physical inactivity, diet and smoking. Genetic factors also play an important role. Apolipoprotein A-I (apoA-I), the main protein of high-density lipoprotein (HDL), is considered to protect against cardiovascular disease and mutations in apoA-I may lead to reduced protective functions of HDL. Despite the fact that many mutations have been described, the putative presence of mutated apoA-I in plasma HDL and the associated effects on atherosclerosis in patients have hardly been studied. Mutated apoA-I might be present in different concentrations depending on the type of mutation. Moreover, carriers may have an altered HDL protein composition that influences the specific anti-atherogenic functions of HDL. A better understanding of expression of mutated apoA-I as well as the expression of other HDL proteins may shed light on the clinical presentation of affected individuals and provide better information on how to HDL is related to CVD. 


\section{Abstract}

\section{Purpose}

Mutations in apolipoprotein A-I may affect plasma HDL-C levels and the risk for cardiovascular disease but little is known about the presence and effects of circulating apoA-I variants. This study investigates whether the apoA-I mutations apoA-I ${ }^{\mathrm{L} 202 \mathrm{P}}$ and apoA-I ${ }^{\mathrm{K} 131 \mathrm{del}}$ are present on plasma HDL particles derived from heterozygote carriers and whether this is associated to changes in HDL protein composition.

\section{Experimental design}

Plasma HDL of heterozygotes for either apoA-I ${ }^{\mathrm{L} 202 \mathrm{P}}$ or apoA- $\mathrm{I}^{\mathrm{K} 131 \mathrm{del}}$ and family controls was isolated using ultracentrifugation. HDL proteins were separated by twodimensional gel electrophoresis (2-DE) and analyzed by MS.

\section{Results}

ApoA-I peptides containing apoA-I ${ }^{\mathrm{L} 202 \mathrm{P}}$ or apoA-I ${ }^{\mathrm{K} 131 \mathrm{del}}$ were identified in HDL from heterozygotes. The apoA-I ${ }^{\mathrm{L} 202 \mathrm{P}}$ mutant peptide was less abundant than wild-type peptide while the apoA-I ${ }^{\mathrm{K} 131 \mathrm{del}}$ mutant peptide was more abundant than wild-type peptide in the heterozygotes. 2-DE analyses indicated that compared to controls, HDL in apoA-I ${ }^{\mathrm{L} 202 \mathrm{P}}$ carriers contained less apoE and more zinc-alpha-2-glycoprotein while HDL from the apoA-I ${ }^{\mathrm{K} 131 \mathrm{del}}$ heterozygotes contained more alpha-1-antitrypsin and transthyretin.

\section{Conclusions and clinical relevance}

Both apoA-I ${ }^{\mathrm{L} 202 \mathrm{P}}$ and apoA-I ${ }^{\mathrm{K} 131 \mathrm{del}}$ were identified in HDL. In heterozygotes, these mutations have markedly differential effects on the concentration of wild-type apoA-I in the circulation, as well as the HDL proteome, both of which might affect the clinical phenotype encountered in the heterozygous carriers. 


\section{Introduction}

Cardiovascular disease (CVD) is the leading cause of death in the industrialized world and has the highest disease burden for affected individuals when calculated as lifeyears lost [1]. The concentration of high-density lipoprotein cholesterol (HDL-C) has been shown to be inversely correlated to risk of CVD [2] and HDL is suggested to have atheroprotective effects by inducing cholesterol efflux from lipid-laden cells in the vascular wall and to reduce inflammatory, oxidative and thrombogenic signals $[3,4]$. Today, there is a major need to find specific functions, protein or lipid compositions of HDL that are determinants of the anti-atherosclerotic capacity of HDL [5].

Apolipoprotein A-I (apoA-I), the main structural protein of HDL, plays a key role in the genesis of HDL through its interaction with ATP-binding cassette A1 (ABCA1). In this process, essentially lipid-free apoA-I acquires free cholesterol and phospholipids resulting in the formation of pre-beta HDL (disc-shaped nascent HDL). As a cofactor of lecithin: cholesterol acyltransferase (LCAT), apoA-I is also essential for cholesteryl ester generation on HDL particles thereby directly affecting HDL maturation, i.e. the generation of larger spherical macromolecules [4]. In addition, apoA-I serves as a ligand for the binding of HDL to Scavenger Receptor Class B-1 (SR-B1) [6,7] thereby mediating cellular cholesterol uptake in target organs like the liver and adrenals.

About 50 different mutations in apoA-I have been described, and of these over 20 have been linked to low levels of HDL-C in the circulation. In general, these 
mutations can be divided into two distinct groups, those that mostly reside within the $\mathrm{N}$-terminal part of the protein and have been associated with amyloidosis and those residing within the central region and believed to affect the LCAT-activity [8]. ApoA-I contains ten helical repeats that, during HDL formation, create a double belt structure with the hydrophobic amino acids towards the lipid center [9]. Helical repeats 6 to 7 and in particular $\operatorname{Arg}^{173}, \operatorname{Arg}^{177}$ and $\operatorname{Arg}^{184}[10]$, and a protrusive loop in residues 183-204 [11], are most likely crucial for the LCAT activation. Thus far only a few of the natural apoA-I mutations identified in humans have been described to be associated with altered/increased CVD risk. In addition, there is almost no information on the concentrations of e.g. the different apoA-I variants in plasma and how this may affect HDL genesis, function and composition.

Two mutations in the central region of full length (including signal and propeptide) apoA-I that are associated with decreased apoA-I and HDL-C levels as well as an increased risk for $\mathrm{CVD}$ are apoA- $\mathrm{I}^{\mathrm{L} 202 \mathrm{P}}$ (previously annotated as apoA-I ${ }^{\mathrm{L} 178 \mathrm{P}}$ excluding signal- and pro-peptide) [12] and apoA-I ${ }^{\mathrm{K} 131 \mathrm{del}}$ (previously annotated as apoA-I ${ }^{\text {K107del }}$, Marburg/Munster-2 variant and apoA-I Helsinki [13,14]. The new annotations follow the international guidelines for describing mutations in the human genome (http://www.hgvs.org/rec.html). The novel apoA-I ${ }^{\mathrm{L} 202 \mathrm{P}}$ mutation was identified in six families and was associated with endothelial dysfunction and increased arterial wall thickness, a surrogate marker of CVD [12]. At that time, the mutant was not detected in plasma and it was assumed that the phenotypic effects were due to the half normal levels of wild-type apoA-I in the circulation of heterozygotes for this mutation. The apoA- $\mathrm{I}^{\mathrm{K} 131 \mathrm{del}}$ mutation on the other hand was shown to be present in Finnish kindred with high incidence of premature CVD but to 
have no effect on LCAT activity in vitro [14]. This mutation has also been found in a subject with intimal amyloidosis deposits combined with extensive atherosclerosis [15]. In the current study, we have investigated the presence of apoA-I ${ }^{\mathrm{L} 202 \mathrm{P}}$ and apoA$\mathrm{I}^{\mathrm{K} 131 \mathrm{del}}$ variants in HDL of heterozygotes using improved MS analyses. In addition, we analyzed their HDL protein profiles.

\section{Materials and Methods}

\subsection{Chemicals}

Endoproteinase Glu-C and 2,5-dihydroxybenzoic acid was purchased from SigmaAldrich (St. Louis, MO, US), IPG Dry strips were obtained from GE Healthcare (Little Chalfont, UK), Sypro Ruby stain was obtained from Bio-Rad (Hercules, CA, US), trypsin was purchased from Promega (Fitchburg, WI, US) and a standard peptide mixture for peptide mass fingerprinting was obtained from Applied Biosystems (Foster City, CA, US).

\subsection{Study population}

Three heterozygotes for the apoA-I ${ }^{\mathrm{L} 202 \mathrm{P}}$ mutation were recruited from a study initially aimed at identifying genes that control HDL-C levels as described previously [12]. Three subjects heterozygous for the apoA- $\mathrm{I}^{\mathrm{K} 131 \mathrm{del}}$ mutation were identified by direct sequencing of the apoA-I gene in subjects with hypoalphalipoproteinemia. For both mutations, three age and gender matched family controls were included. Informed consent was obtained from all subjects for plasma sampling, storage, genetic and proteomic analysis, and vascular tests, under a protocol approved by the ethics committee of the Academic Medical Center in Amsterdam. Past medical history, 
presence of cardiovascular risk factors, use of medication, and information on geographic origin of the probands were assessed by questionnaires.

\subsection{Isolation of HDL}

Fasting blood was drawn (in EDTA containing tubes) from the subjects and centrifuged at $3000 \mathrm{~g}$ for 20 minutes. The plasma was separated and stored at $-80^{\circ} \mathrm{C}$ until further analysis. HDL was isolated as previously described [16]. In short, plasma was mixed with $5 \%$ sucrose and $10 \mathrm{mg} / \mathrm{mL}$ EDTA and gently overlayered with a $\mathrm{KBr} /$ phosphate buffer solution (density $1.063 \mathrm{~g} / \mathrm{mL}$ ). Ultracentrifugation was performed at $290000 \mathrm{~g}$ at $15^{\circ} \mathrm{C}$ for 4 hours. HDL was collected from the middle of the tube and the fraction was mixed with a $\mathrm{KBr} / \mathrm{phosphate}$ buffer (density $1.24 \mathrm{~g} / \mathrm{mL}$ ) and a second round of ultracentrifugation was performed under the same conditions for $2 \mathrm{~h}$. HDL was collected from the top of the tubes and desalted. Protein concentration was measured with the Bio-Rad protein assay (Bio-Rad) and samples were lyophilized.

\subsection{Two-dimensional gel electrophoresis (2-DE)}

The 2-DE was performed as described earlier [16]. In short, lyophilized samples were reconstituted in a 2-DE sample solution [17] and $300 \mu \mathrm{g}$ of total protein was isoelectrically focused in IPG Dry strip pH 3-10NL for 53000 Vhrs. The second dimension was done by transferring the IPG strip to a homogenous $14 \%$ acrylamide gel on gel bond and running the electrophoresis overnight. Separated proteins were detected with Sypro Ruby and visualized with a Versadoc system (Bio-Rad) and PDQuest software (Bio-Rad). Gel spots were matched and intensities were evaluated by relative densitometric quantification. 


\subsection{In gel digestion with Glu-C}

Protein spots were visualized with a blue light transluminator (DR-180 B; Clara Chemical Research, Denver, CO, USA) and excised from the 2-DE gel. Excised gel pieces were washed with $50 \%$ acetonitrile/25 $\mathrm{mM}$ ammonium bicarbonate followed by $100 \%$ acetonitrile before being dried using a SpeedVac vacuum concentration system. Gel pieces were rehydrated with endoproteinase Glu-C $(20 \mu \mathrm{g} / \mathrm{mL})$ in $25 \mathrm{mM}$ ammonium bicarbonate and proteins were in-gel digested by incubating at $37^{\circ} \mathrm{C}$ overnight. The supernatant was transferred to a new tube while peptides were further extracted by incubation in $50 \%$ acetonitrile/5\% TFA for $5 \mathrm{~h}$ on a shaker. Supernatants were pooled and dried by SpeedVac centrifugation. Dried peptides were reconstituted in 5 ul $0.1 \%$ TFA.

\subsection{MS analysis}

Proteins were first analyzed by peptide mass fingerprinting. Reconstituted peptides were mixed 1:1 with 2,5-dihydroxybenzoic acid (DHB) in $70 \%$ acetonitrile/0.3\% TFA and spotted on a stainless steel target plate. Peptide masses were analyzed by MALDI-TOF MS (Voyager DE-PRO, Applied Bio Systems) set on reflector mode and positive ionization. Spectra obtained were processed in Data Explorer ${ }^{\mathrm{TM}}$ V4.0 (Applied Biosystems). External calibration using a standard peptide mixture and internal calibration using Glu-C autocatalytic peaks (m/z 1223.70, 1374.75, 2549.23) was done prior to database search using ProteinProspector (University of San Francisco). Verification of the mutant peptides was done by peptide sequencing using electrospray ionization tandem MS (ESI-Q-TOF/MS) on a hybrid spectrometer QSTAR Pulsar I (Applied Biosystems) equipped with a nano-electrospray ion source (MDS Protana, Odense, Denmark). Peptides reconstituted in $0.1 \%$ TFA were desalted by the use of ZipTips (Millipore, Billerica, MA, US) and loaded into an off-line 
nanoelectrospray capillary. A spray voltage of $0.9 \mathrm{kV}$ was used to ionize the peptides. Collision-induced dissociation of selected precursor ions was performed with manual control of collision energy during spectrum acquisition. Peaks corresponding to wildtype and mutant peptides were selected and fragmented for sequencing. Fragmentation spectra were analyzed by manual interpretation in Analyst ${ }^{\mathrm{TM}}$ QS software (Applied Biosystems). Further validation of apoA-I ${ }^{\mathrm{L} 202 \mathrm{P}}$ was done by nanoflow HPLC system (EASY-nLC; Proxeon, Bruker Daltronics) combined with the mass spectrometer HCTUltra PTM Discovery (Bruker Daltronics). Proteins were separated using a $100 \mathrm{~mm} \times 75 \mu \mathrm{m} \mathrm{C} 18$ column at a flow rate of $300 \mathrm{~nL} / \mathrm{min}$. The gradient solutions consisted of $0.1 \%$ formic acid in water (solution A) and $0.1 \%$ formic acid in acetonitrile (solution B) and proteins were separated using a two-step gradient of $0 \%$ to $35 \%$ solution B during 27 minutes followed by a gradient to $100 \%$ solution B during 10 minutes. Capillary temperature and voltage were set to $250^{\circ} \mathrm{C}$ and $150 \mathrm{~V}$ respectively with a gas flow of $6 \mathrm{~L} / \mathrm{min}$. Spectrum were obtained between $\mathrm{m} / \mathrm{z} 100$ and 1500 followed by automated online tandem MS by electron-transfer dissociation. Data was processed in DataAnalysis 3.4 (Bruker Daltronics).

\section{Results}

\subsection{Identification of apoA-I ${ }^{L 202 P}$ in $\mathrm{HDL}$}

In line with the previous report [12], apoA-I ${ }^{\mathrm{L} 202 \mathrm{P}}$ heterozygotes were characterized by an approximate $50 \%$ reduction in plasma apoA-I levels and an approximate $60 \%$ decrease of HDL-C compared with controls (Table I). HDL was isolated from plasma, proteins were separated with 2-DE and apoA-I was further subjected to in-gel digestion. A substitution of leucine for proline does not result in a charge difference, 
and as a consequence, the apoA-I variant apoA-I ${ }^{\mathrm{L} 202 \mathrm{P}}$ and wild-type A-I were expected to be located in the same spot on 2-DE gels in case the mutant was present in plasma HDL isolated through ultracentrifugation. To investigate if the mutated apoA-I form was present in plasma and for optimal detection of both wild-type and mutant peptide including residue 202, the samples were digested with the endoproteinase Glu-C that cleaves peptide bonds C-terminally of glutamic acid. The digests were initially analyzed with MALDI-TOF MS. In the controls, only the peptide mass corresponding to the wild-type peptide (m/z 1225.8) was found (Figure 1A). In contrast, a peptide mass of $\mathrm{m} / \mathrm{z} 1209.7$ corresponding to sequence position 194-203 but with a proline instead of a leucine was found in all three heterozygotes besides the peptide mass peak corresponding to the wild-type peptide (m/z 1225.8) (Figure 1B). The site of the mutation was verified by sequencing on ESI-Q-TOF MS (Figure 1C-E) and with nLC-MS/MS (supplemental Figure 1). Two triply charged peptides (m/z 403.9 and 409.3) corresponding to $\mathrm{m} / \mathrm{z} 1209$ and 1225, respectively, were selected for fragmentation. As shown in figure 1D, the sequence of $\mathrm{m} / \mathrm{z} 403.9$, LRQRLAARPE, confirmed a proline at position 202 in apoA-I. The sequence of $\mathrm{m} / \mathrm{z}$ 409.3, LRQRLAARLE, corresponded to the wild-type peptide (Figure 1E). As shown in figures $1 \mathrm{~B}$ and $1 \mathrm{C}$, the intensity of the mutant peptide peak was about $1 / 3$ of the wild-type peptide peak in the heterozygotes. Relative abundance obtained by peak area ratio determinations (mutant peptide/wild-type peptide) in the MALDI-TOF analyses showed that the expression of the mutant peptide was very similar in the three heterozygotes with a mean of $31.2 \%$ of wild-type (ranged from 29.3 to $33.5 \%$ ). Essentially the same values (30.3\%) were obtained when using ESI-Q-TOF MS as well as when peptides were separated by $\mathrm{C} 18$ reverse phase chromatography using nano-flow HPLC system and analyzed by electrospray ionization ion trap (29.8\%), 
further confirming our initial results. In addition, peak area determinations of a separate major peak (m/z 1252.7) from apoA-I, corresponding to a peptide not at the site of the mutation, showed very similar values between the wild-type individuals and heterozygotes (supplemental Table 1). In agreement with our results the ratio of the mutant peptide and this loading control peptide was also about $1 / 3$ compared to the ratio of the wild-type peptide and this peptide in the heterozygotes (supplemental Table 1).

\subsection{Analyses of apoA-I ${ }^{\mathrm{K} 131 \mathrm{del}}$ in HDL}

In contrast to carriers of the apoA-I ${ }^{\mathrm{L} 202 \mathrm{P}}$ mutation, the heterozygotes for apoA-I ${ }^{\mathrm{K} 131 \mathrm{del}}$ did not have lower apoA-I levels than controls (Table 1). However, they did show a $30 \%$ reduction of HDL-C compared to controls. In line with a deletion of lysine, the 2-DE revealed a negative charge shift in the apoA-I isoform pattern with an additional acidic isoform in the heterozygotes that was not found in the controls (Figure 2). ApoA-I was then subjected to in-gel digestion with Glu-C and analyzed by MALDITOF MS. The peak m/z 2379.2, corresponding to the wild-type peptide (position 117135) was detected in both controls and heterozygotes (Figure 3A and 3B) while the peak $\mathrm{m} / \mathrm{z}$ 2251.1, corresponding to the mutant peptide (position 117-134) was only detected in the three heterozygotes (Figure 3B). The site of the mutation was verified by MS/MS (Figure 3C-E). The triple charged peaks of m/z 793.7 and m/z 751.1, corresponding to $\mathrm{m} / \mathrm{z} 2379.2$ and 2251.1, respectively, were chosen for fragmentation and peptide sequencing. The sequence for the $\mathrm{m} / \mathrm{z} 793.7$ corresponded to the wildtype peptide VKAKVQPYLDDFQKKWQEE (Figure 3D) while $\mathrm{m} / \mathrm{z} \quad 751.1$ corresponded to the mutant peptide VKAKVQPYLDDFQKWQEE (Figure 3E). In accordance with 2-DE pattern, the proportion of the mutant peptide was very different 
in the apoA-I isoforms. The most acidic isoform analyzed contained almost only the mutant peptide (>99\%) and the proportion of the mutant gradually decreased with increased pI. The most basic isoform analyzed contained almost only the wild-type peptide $(>95 \%)$. To estimate total relative abundance of the mutant and wild-type peptide, digests from all apoA-I isoforms were pooled and analyzed by MALDI-TOF MS. As illustrated in figure $3 \mathrm{~B}$ and $3 \mathrm{C}$, peak area ratio determinations (mutant peptide/wild-type peptide) showed that interestingly the mutant peptide was about 3fold more abundant than the wild-type peptide in HDL from the heterozygotes (ranged from 200 to $480 \%$ ). The overall amounts of apoA-I in the analyses were about the same in heterozygotes and wild-type controls, as judged by peak area determinations of a major peak $(\mathrm{m} / \mathrm{z}$ 1252.7) not at the site of the mutation (supplemental Table 1). In addition, the ratio of the mutant peptide and this loading control peptide was about 3 times the ratio of the wild-type peptide and the control peptide in the heterozygotes (supplemental Table 1).

\subsection{Protein profiling of HDL from apo $A-I^{L 202 P}$ and apoA-I ${ }^{\mathrm{K} 131 \mathrm{del}}$ heterozygotes}

Analyses by 2-DE/MS showed alterations in the protein distribution patterns in HDL from the heterozygotes (Table 2, supplemental Figure 2 and supplemental Table 2). Besides less apoA-I, HDL of apoA-I ${ }^{\mathrm{L} 202 \mathrm{P}}$, heterozygotes contained less apoE and more zinc- $\alpha$-2-glycoprotein than the controls. On the other hand, HDL of heterozygotes for the apoAI ${ }^{\mathrm{K} 131 \text { del }}$ mutation had increased distribution of $\alpha$-1-antitrypsin and transthyretin. 


\section{Discussion}

We have previously reported that heterozygous carriers of the apoA- $\mathrm{I}^{\mathrm{L} 202 \mathrm{P}}$ are characterized by low HDL-C, increased arterial wall thickness, endothelial dysfunction and a 24-fold increase in risk for CVD in heterozygotes compared to family controls [12]. Moreover, heterozygous carriers have reduced tissue cholesterol efflux [18] and their HDL appears to be functionally impaired with decreased antioxidant and anti-inflammatory potential [19]. In the initial study, apoA-I ${ }^{\mathrm{L} 202 \mathrm{P}}$ was described not to be present in plasma of heterozygotes. The hypothesis that the mutated apoA-I was not expressed was strengthened by the observation of half normal levels of apoA-I in carriers [12]. In the current study, however, we have been able to clearly identify the mutated protein in HDL from the heterozygotes. By using Glu C instead of trypsin, larger peptides were obtained that facilitated the detection of the wild-type and the mutant peptide in the same sample. Our MS results also indicate that the mutated form of apoA-I is less abundant than the wild-type form and comprises approximately $25 \%$ of the total apoA-I in HDL. Leu ${ }^{202}$ is located in $\alpha$-helix 7 and, in total, human native apoA-I contain ten helical repeats and ten prolines [20]. Except for three prolines located N-terminally, the others separate seven of the helical repeats and are important for the correct bending of the apoA-I structure to allow the protein to bind the periphery of a spherical HDL particle [9]. Helices 7/8 do not contains an intermediate proline and experiments with mouse/human chimeric apoA-I indicate that introduction of a proline affects the binding of apoA-I to different sized

HDL subclasses [21]. Therefore, it is tempting to speculate that replacement of Leu ${ }^{202}$ with proline in $\alpha$-helix 7 may introduce an additional structural bend, which impairs the formation of native HDL. However, structural studies of apoA-I and HDL in relation to the mutation are needed to address whether this holds true. 
Interestingly, the data obtained while studying apoA-I in carriers of the apoA-I ${ }^{\mathrm{K} 131 \mathrm{del}}$ show very different results: In sharp contrast to apoA-I ${ }^{\mathrm{L} 202 \mathrm{P}}$, the apoA-I ${ }^{\mathrm{K} 131 \text { del }}$ was shown not to affect total apoA-I in the circulation and was more abundant than wildtype apoA-I comprising about $75 \%$ of total apoA-I in HDL of carriers. The relative abundance of the two different mutants and wild-type apoA-I was based on assessments of the mutant/wild-type peptide peak area ratios. In this respect, other factors that may affect the peak intensities should be considered such as the degree of ionization of wild-type and mutated peptides. However, substitution of a leucine with a proline would most likely create an increased signal [22] while loss of lysine a lower signal in MS. Since we observed the opposite, i.e. less of the mutated peptide than the wild-type peptide in the apoA-I ${ }^{\mathrm{L} 202 \mathrm{P}}$ heterozygotes and more of the mutated peptide than the wild-type peptide in the apoA-I ${ }^{\mathrm{K} 131 \mathrm{del}}$ heterozygotes, it is therefore more likely that we are underestimating the difference between the two mutations rather than overestimating it. By analyzing the two apoA-I variants separated with 2DE, we also diminished possible bias caused by ion-suppression due to interference of other proteins in the analyses. In addition, essential the same results were obtained when normalizing the mutant peptide and wild-type peptide peak areas to the peak area of a major internal control peptide, not at the site of the mutation. Previously, a similar approach as we present in the current study has been applied to determine plasma ratio of wild-type/apoAI ${ }^{\mathrm{L} 159 \mathrm{R}}$ (apoA-I $\mathrm{I}_{\text {Fin }}$ ) $[23,24]$ and to study myosin mutations in relation to cardiomyopathy [25]. Thus, differential quantitation of wildtype and mutant proteins with MS is an interesting new tool to study molecular and biochemical effects of mutations in heterozygotes and this approach has the potential to be more informative than traditional protein assays. Although validated and 
expanded analyses of more subjects are needed, our analyses do display remarkably different ratios of mutant/wild-type apoA-I in HDL from the two groups of heterozygotes; ratio $>1$ in apoA-I ${ }^{\mathrm{K} 131 \mathrm{del}}$ and ratio $<1$ in apoA-I $\mathrm{I}^{\mathrm{L} 202 \mathrm{P}}$. Possible clinical effects of these results are as yet unclear but interestingly indicate that apoA-I ${ }^{\mathrm{K} 131 \mathrm{del}}$ could be more stable than the wild-type variant.

The analyses of HDL protein composition showed significantly less apoE and more zinc- $\alpha$-2-glycoprotein in the apoA-I ${ }^{\mathrm{L} 202 \mathrm{P}}$ heterozygotes and significantly more $\alpha-1$ antitrypsin and transthyretin in the apoA- $\mathrm{I}^{\mathrm{K} 131 \mathrm{del}}$ heterozygotes, compared to controls. A reduction of apoE, another protein with atheroprotective properties [26], may be an additional risk factor for CVD in apoA- $\mathrm{I}^{\mathrm{L} 202 \mathrm{P}}$ heterozygotes. The function of zinc- $\alpha-2-$ glycoprotein is largely unknown but it is regarded as a novel adipokine involved in immune responses [27]. The protein has also been suggested as a biomarker for CVD as the levels are positively associated with C-reactive protein and triglyceride levels, and negatively associated with HDL-C levels in obese subjects with metabolic syndrome [28].

Increased expression of the serine protease inhibitor $\alpha$-1-antitrypsin in HDL of apoA$\mathrm{I}^{\mathrm{K} 131 \mathrm{del}}$ heterozygous carriers may reflect increased inflammation in the vascular wall since $\alpha-1$-antitrypsin is a well-known acute phase reactant with anti-inflammatory and immuno-modulatory properties that binds to HDL [29]. Furthermore, it may be involved in several different inflammatory diseases, including atherosclerosis [30-32]. We also found more of transthyretin in HDL of carriers with this mutation. Transthyretin is an abundant serum protein that can associate with HDL and LDL [33]. There is no data on a possible function of transthyretin in lipoproteins but the 
protein has been described as an apoA-I interacting cryptic protease that increases apoA-I amyloidogenicity [34]. In line, the apoA-I ${ }^{\mathrm{K} 131 \mathrm{del}}$ mutation has been found in intimal amyloidosis deposits combined with extensive atherosclerosis [15]. Previous proteomic studies have suggested that sulfated transthyretin is a biomarker for myocardial infarction [35] while others showed an inverse correlation between transthyretin and cardiovascular risk [36].

In our study, we have not addressed whether the mutations in apoA-I studied affect HDL cholesterol levels in plasma through anabolic or catabolic processes. Such studies of fractional synthetic and catabolic rates can be performed by gene transfer of the apoA-I mutants in apoA-I deficient backgrounds or transgenic mice of these mutants in apoA-I deficient backgrounds. However, patients suffering from complete apoA-I deficiency are characterized by pronounced HDL deficiency $[37,38]$. On the other hand, overexpression of apoA-I causes increased levels of HDL cholesterol in mice. Although, we have not identified homozygotes for the L202P and K131del defects, it is safe to assume that these mutations also affect the production of small nascent HDL and that the mutated apoA-I's are likely poor substrates for lipidation by ABCA1. Of note in this regard is that ABCAI deficiency (a disorder also known as Tangier Disease) is in contrast characterized by hypercatabolism of small (abnormal) nascent HDL which also leads to HDL deficiency.

The inverse correlation between HDL-C and CVD is well established and many studies have shown that a decrease in HDL-C is associated with an increased risk of CVD. However, HDL-C levels are influenced by many factors that also influence CVD risk [5, 39]. Interestingly, Sbrana et al recently concluded that also patients with 
elevated HDL-C may develop CVD but later in life [40]. In addition, a recent Mendelian Randomization study provides strong evidence that genetic mechanisms raising HDL-C do not always confer lower risk of myocardial infarction [41]. This study adds to the current confusion on the HDL hypothesis, i.e. whether HDL-C is causally related to atherosclerosis or not [42]. In the case of apoA-I, however, most studies are consistent with a loss of apoA-I being atherogenic, while an increase in apoA-I is atheroprotective $[2,5]$. While the association between apoA-I mutations and risk of atherosclerosis is more complex, the current study shows that in heterozygotes for apoA-I mutations, one can find unanticipated effect of the mutants on the HDL proteome. Our analyses of two apoA-I mutations in heterozygotes indicate an unequal expression of wild-type and mutant protein in HDL. This can be caused by a multitude of co- or post-translational events such as different mRNA-expression and processing as well as different protein-folding, -secretion, -catabolism and lipid binding. In any case, the two mutations interestingly contrasted each other; in apoA$\mathrm{I}^{\mathrm{L} 202 \mathrm{P}}$ the mutated protein was less abundant than the wild-type protein while in apoA$\mathrm{I}^{\mathrm{K} 131 \mathrm{del}}$ the mutated protein was more abundant than the wild-type protein. In addition, we found that the mutants studied also distinctly affected the concentrations of other proteins in the HDL fraction.

\section{Acknowledgements}

This work was funded by EU's Sixth Framework Program: FP6-2005 No. 037631 (HDLomics), the Research Council of South East Sweden (FORSS-3755), County Council of Östergötland (C-ALF) and Faculty of Health Sciences in Linköping. Drs Hovingh and Holleboom are supported by Veni grants (project numbers 91612122 
and 91613031, respectively) from NWO. Dr. Kuivenhoven is supported by Fondation LeDucq (Transatlantic Network, 2009-2014), the Netherlands CardioVascular Research Initiative (CVON2011-19; Genius) and the European Union (Resolve: FP7305707; TransCard: FP7-603091-2).

\section{Competing interests}

No competing interests.

\section{References}

[1] Lozano, R., Naghavi, M., Foreman, K., Lim, S. et al., Global and regional mortality from 235 causes of death for 20 age groups in 1990 to 2010: a systematic analysis for the Global Burden of Disease Study 2010. Lancet 2012, 380, 2095-2128. [2] Emerging risk factors collaboration, Major lipids, apolipoproteins, and risk of vascular disease. JAMA. 2009, 302, 1993-2000.

[3] Barter, P., HDL-C: Role as a risk modifier. Atheroscler. Supp. 2011, 12, 267-270.

[4] Davidson, M., Toth, P., High-density lipoprotein metabolism: Potential therapeutic targets. Am. J. Card. 2007, 100, 32N-40N

[5] Besler, C., Lusher, TF., Landmesser, U., Molecular mechanisms of vascular effects of High-density lipoprotein: alterations in cardiovascular disease. EMBO Mol. Med. 2012, 4, 251-268.

[6] Xu, S., Laccotripe, M., Huang, X., Rigotti, A. et al., Apolipoproteins of HDL can directly mediate binding to the scavenger receptor SR-BI, an HDL receptor that mediates selective lipid uptake. J. Lipid Res. 1997, 3, 1289-1298. 
[7] Liu, T., Krieger, M., Kan, H., Zannis, V., The effects of mutations in helices 4 and 6 of apoA-I on scavenger receptor class B type 1 (SR-B1)-mediated cholesterol efflux suggest that formation of a productive complex between reconstituted high density lipoprotein and SR-B1 is required for efficient lipid transport. J. Biol. Chem. 2002, $277,21576-21584$.

[8] Sorci-Thomas, M., Thomas, M., The Effects of altered Apolipoprotein A-I structure on plasma HDL concentration. Trends Cardiovasc. Med. 2002, 12, 121-128. [9] Thomas, M., Bhat, S, Sorci-Thomas, M., Three-dimensional models of HDL ApoA-I: implications for its assembly and function. J. Lipid Res. 2008, 49, 18751883.

[10] Roosbeek, S., Vanloo, B., Duverger, N., Caster, H. et al., Three arginine residues in apolipoprotein A-I are critical for activation of lecithin:cholesterol acyltransferase. J. Lipid Res. 2001, 42, 31-40.

[11] Wu, Z., Wagner, M., Zheng, L., Parks, J. et al., The refined structure of nascent HDL reveals a key functional domain for particle maturation and dysfunction. Nat. Struct. Mol. Biol. 2007, 14, 861-868.

[12] Hovingh, G., Brownlie, A., Bisoendial, R., Dube, M. et al., A novel ApoA-I mutation (L178P) leads to endothelial dysfunction, increased arterial wall thickness, and premature coronary artery disease. J Am Coll Cardiol. 2004, 44, 1429-1435.

[13] Rall, S., Weisgraber, K., Mahley, R., Ogawa, Y. et al., Abnormal Lecithin:cholesterol acyltransferase activation by a human Apolipoprotein A-I variant in which a single lysine residue is deleted. J. Biol. Chem. 1984, 259, 10063-10070.

[14] Tilly-Kiesi, M., Qiuping, Z., Ehnholm, S., Kahri, J. et al., ApoA-IHelsinki (Lys107 $\rightarrow 0$ ) associated with reduced HDL cholesterol and LpA-I:A-II deficiency. Arterioscler. Thromb. Vasc. Biol. 1995, 15, 1294-1306. 
[15] Amarzguioui, M., Mucchiano, G., Häggqvist, B., Westermark, P. et al., Extensive intimal Apolipoprotein A1-derived amyloid deposits in a patient with an Apolipoprotein A1 mutation. Biochem. Biophys. Res. Commun. 1998, 242, 534-539.

[16] Karlsson, H., Leandersson, P., Tagesson, C., Lindahl, M., Lipoproteomics II: mapping of proteins in high-density lipoprotein using two-dimensional gel electrophoresis and mass spectrometry. Proteomics 2005, 5, 1431-1445.

[17] Görg, A., Obermaier, C., Boguth, G., Harder, A. et al., The current state of twodimensional electrophoresis with immobilized pH gradients. Electrophoresis 2000, 21, 1037-1053.

[18] Holleboom, A., Jakulj, L., Franssen, R., Decaris, J. et al., In vivo cholesterol efflux is reduced in carriers of a mutation in APOA1. J. Lipid. Res. 2013, Epub ahead of print

[19] Daniil, G., Phedonos, A., Holleboom, A.G, Motazacker, M. et al., Characterization of antioxidant/anti-inflammatory properties and apoA-I-containing subpopulations of HDL from family subjects with monogenic low HDL disorders. Clin. Chim. Acta. 2011, 412, 1213-1220.

[20] Borhani, D., Rogers, D., Engler, J., Brouilette, C., Crystal structure of truncated human apolipoprotein A-I suggests a lipid-bound conformation. Proc. Natl. Acad. Sci. U S A. 1997, 94, 12291-12296.

[21] Reschly, E., Sorci-Thomas, M., Davidson, S., Meredith, S. et al., Apolipoprotein A-I $\alpha$-helices 7 and 8 modulate high density lipoprotein subclass distribution. J. Biol. Chem. 2002, 277, 9645-9654.

[22] Cech, N., Enke, C., Practical implications of some recent studies in electrospray ionization fundamentals. Mass. Spectrom. Rev. 2001, 20, 362-387. 
[23] Owen, J., Bharadwaj, M., Thomas, M., Bhat, S. et al., Ratio determination of plasma wild-type and L159R apoA-I using mass spectrometry: tools for studying

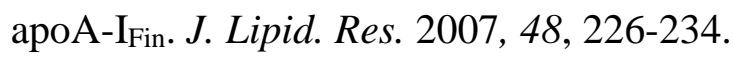

[24] Sorci-Thomas, M., Zabalawi, M., Bharadwaj, M., Wilhelm, A. et al., Dysfunctional HDL containing L159R ApoA-I leads to exacerbation of atherosclerosis in hyperlipidemic mice. Biochim. Biophys. Acta. 2012, 1821, 502-512 [25] Nier, V., Schultz, I., Brenner, B., Forssmann, W., Raida, M., Variability in the ratio of mutant to wild-type myosin heavy chain present in the soleus muscle of patients with familial hypertrophic cardiomyopathy. A new approach for the quantication of mutant to wild-type protein. FEBS Lett. 1999, 461, 246-252.

[26] Kypreos, K., Zannis, V., Pathway of biogenesis of apolipoprotein E-containing HDL in vivo with the participation of ABCA1 and LCAT. Biochem. J. 2007, 403, 359-367.

[27] Hassan, M., Waheed, A., Yadav, S., Singh, TP., Ahmad, F., Zinc a2glycoprotein: a multidisciplinary protein. Mol. Cancer Res. 2008, 6, 892-906.

[28] Yeung, D., Lam, K., Wang, Y., Tso, A., Xu, A., Serum Zinc-2-glycoprotein correlates with adiposity, triglycerides, and the key components of the metabolic syndrome in chinese subjects. J. Clin. Endocrinol. Metab. 2009, 94, 2531-2536.

[29] Vaisar, T., Pennathur, S., Green, P., Gharib, S. et al., Shotgun proteomics implicates protease inhibition and complement activation in the anti-inflammatory properties of HDL. J. Clin. Invest. 2007, 117, 746-756.

[30] Janciauskiene, S., Bais, R., Koczulla, R., Vogelmeier, C. et al., The discovery of a1-antitrypsin and its role in health and disease. Respir. Med. 2011, 105, 1129-1139. 
[31] Talmud, P., Martin, S., Steiner, G., Flavell, D. et al., Progression of atherosclerosis is associated with variation in the a1-antitrypsin gene. Arterioscler. Thromb. Vasc. Biol. 2003, 23, 644-649.

[32] Donners, M., Verluyten, M., Bouwman, F., Mariman, E. et al., Proteomic analysis of differential protein expression in human atherosclerotic plaque progression. J. Pathol. 2005, 206, 39-45.

[33] Karlsson, H., Mortstedt, H., Lindqvist, H., Tagesson, C., Lindahl, M., Protein profiling of low-density lipoprotein from obese subjects. Proteomics Clin. Appl. 2009, $3,663-671$.

[34] Liz, M., Gomes, C., Saraiva, M., Sousa, M., ApoA-I cleaved by transthyretin has reduced ability to promote cholesterol efflux and increased amyloidogenicity. J. Lipid Res. 2007, 48, 2385-2395.

[35] Kiernan, U., Nedelkov, D., Nelson, R., Multiplexed mass spectrometric immunoassay in biomarker research. A novel approach to the determination of a myocardial infarct. J. Proteome Res. 2006, 5, 2928-2934.

[36] Cubedo, J., Padró, T., Alonso, R., Cinca, J. et al., Differential proteomic distribution of TTR (pre-albumin) forms in serum and HDL of patients with high cardiovascular risk. Atherosclerosis 2012, 222, 263-269.

[37] Schaefer, E., Heaton, W., Wetzel, M., Brewer H., Plasma apolipoprotein A-1 absence associated with marked reduction of high density lipoproteins and premature coronary artery disease. Arterioscler. Thromb. Vasc. Biol. 1982, 2, 16-26

[38] Wada, M., Iso, T., Asztalos, B., Takama, N. et al., Marked high density lipoprotein deficiency due to apolipoprotein A-I Tomioka (codon 138 deletion). Atherosclerosis 2009, 207, 157-161 
[39] Vergeer, M., Holleboom, A., Kastelein, J., Kuivenhooven, J., The HDL hypothesis: does high-density lipoprotein protect from atherosclerosis?. J. Lipid. Res. 2010, 51, 2058-2073.

[40] Sbrana, F., Puntoni, M., Bigazzi, F., Landi, P. et al., High density lipoprotein cholesterol in coronary artery disease: when higher means later. J. Atheroscler. Thromb. 2013, 20, 23-31.

[41] Voight, B., Peloso, G., Orho-Melander, M., Frikke-Schmidt, R. et al., Plasma HDL cholesterol and risk of myocardial infarction: a mendelian randomization study. Lancet 2012, 380, 572-580.

[42] Rader, D., Tall, A., The not so simple HDL story: Is it time to revise the HDL cholesterol hypothesis. Nat. Med. 2013, 18, 1344-1346. 


\section{Legends to figures}

Figure 1 - MS and MS/MS analysis of mutant apoA-I ${ }^{\mathrm{L202P}}$

Peptides after Glu-C digestion of apoA-I from a control (A) and an apoA-I ${ }^{\mathrm{L} 202 \mathrm{P}}$ heterozygote (B-E) analyzed by MALDI-TOF MS (A and B) and ESI-Q-TOF MS (CE). Left: The $\mathrm{m} / \mathrm{z}(\mathrm{M}+\mathrm{H})^{+}$of the peaks corresponding to wild-type LRQRLAARLE and mutant LRQRLAARPE peptides are indicated. Right: Mass spectrum of the triply charged peptides with $\mathrm{m} / \mathrm{z} 403.9$ and 409.3 (C), corresponding to the mutant LRQRLAARPE and wild-type LRQRLAARLE peptides, respectively. Below are the collision-induced fragmentation spectra of the 403.9 (D) and 409.3 (E) peptide ions. The $b$ (N-terminal) and $y$ (C-terminal) fragment ions are indicated in the spectra and in the corresponding peptide sequences. Some intense internal fragment signals; P, RP and AAR in spectrum D and L and AAR in spectrum $\mathrm{E}$ are also marked.

\section{Figure 2 - Isoelectric shift in apoA-I ${ }^{\mathrm{K} 131 \mathrm{del}}$ isoform}

2-DE separation of apoA-I isoforms in HDL from a wild-type (WT) and a heterozygote $(\mathrm{Het})$ for the apoA-I ${ }^{\mathrm{K} 131 \mathrm{del}}$ mutation. Arrow indicates the additional isoform in the heterozygote, compared to the control, caused by a shift in isoelectric point from loss of a lysine. The isoform marked with 1 contains mutant protein, isoform 2 contains a mixture of mutant as well as wild-type protein and isoform 3 contains wild-type protein.

\section{Figure 3 - MS and MS/MS analysis of mutant apoA-I ${ }^{\mathrm{K} 131 \mathrm{del}}$}

Peptides after Glu C digestion of five pooled apoA-I isoforms from a control (A, Cupper and D) and apoA-I ${ }^{\mathrm{K} 131 \mathrm{del}}$ heterozygote (B, C-lower and E) analyzed by MALDITOF MS (A and B) and ESI-Q-TOF MS (C-E). Left: The m/z $(\mathrm{M}+\mathrm{H})^{+}$of the peaks 
corresponding to mutant VKAKVQPYLDDFQKWQEE and wild-type VKAKVQPYLDDFQKKWQEE peptides are indicated. Right: Mass spectrum of the quadruple charged peptides with $\mathrm{m} / \mathrm{z} 595.56$ and 563.54 (C), corresponding to the wild-type VKAKVQPYLDDFQKKWQEE and mutant VKAKVQPYLDDFQKWQEE peptides, respectively. Below are the collisioninduced fragmentation spectra of triple charged 793.7 (D) and 751.1 (E) peptide ions. The $b(\mathrm{~N}$-terminal) and $y(\mathrm{C}$-terminal) fragment ions are indicated in the spectra and in the corresponding peptide sequences. Intense internal fragment signals; K or Q are marked in both spectra. 
Table 1 - Lipid and lipoprotein levels in apoA-IL202P and apoA-I ${ }^{\mathrm{K} 131 \mathrm{del}}$ heterozygotes and family controls

Data from three apoA-I ${ }^{\mathrm{L} 202 \mathrm{P}}$ heterozygotes, three apoA-I ${ }^{\mathrm{K} 131 \mathrm{del}}$ heterozygotes and three family controls for each mutation. Median (range). $*=p \leq 0.05$ vs controls (Mann-Whitney U-test).

\begin{tabular}{|ccccc|}
\hline & $\begin{array}{c}\text { apoA-I } \\
\text { Controls }\end{array}$ & $\begin{array}{c}\text { apoA-I } \\
\text { Heterozygotes }^{\text {L202P }}\end{array}$ & $\begin{array}{c}\text { apoA-I } \\
\text { Controls }\end{array}$ & $\begin{array}{c}\text { apoA-I }^{\text {K131del }} \\
\text { Heterozygotes }\end{array}$ \\
\hline $\begin{array}{c}\text { Total Cholesterol } \\
(\mathrm{mmol} / \mathrm{l})\end{array}$ & $4.4(4.0-5.3)$ & $3.5(3.4-4.0)$ & $4.2(4.1-4.5)$ & $3.8(3.0-5.3)$ \\
HDL-C (mmol/l) & $1.2(1.1-1.2)$ & $0.4(0.2-0.9)^{*}$ & $0.9(0.8-0.9)$ & $0.6(0.3-0.8)$ \\
Triglycerides & $1.1(0.8-1.4)$ & $1.0(0.6-1.6)$ & $1.4(1.0-2.1)$ & $2.3(1.7-2.3)$ \\
$(\mathrm{mmol} / \mathrm{l})$ & & & & \\
ApoA-I (mg/dl) & $167(148-174)$ & $83(50-126)^{*}$ & $113(102-119)$ & $109(84-144)$ \\
ApoB (mg/dl) & $91(85-114)$ & $82(82-93)$ & $88(79-95)$ & $85(69-113)$ \\
\hline
\end{tabular}


Table 2 - Protein differences in HDL from the apoA-I ${ }^{\mathrm{L} 202 \mathrm{P}}$ and apoA-I ${ }^{\mathrm{K} 131 \mathrm{del}}$ heterozygotes

Proteins were analyzed with 2-DE stained with Sypro Ruby fluorescence. Values are median (range) expressed as $\%$ of total gel image fluorescence. $*=\mathrm{p} \leq 0.05$ vs controls (Mann-Whitney U-test)

\begin{tabular}{|cccc|}
\hline Protein & kDa/pI & Controls & Heterozygotes \\
\hline apoA-I $I^{L 202 P}$ & & & \\
ApoE & $32 / 5.4-30 / 5.7$ & $2.6(1.2-7.5)$ & $0.8(0.4-0.8)^{*}$ \\
Zinc-alpha-2-glycoprotein & $45 / 4.0$ & $<0.1(<0.1-0.1)$ & $0.7(0.7-1.2)^{*}$ \\
apoA-I $I^{K 13 l d e l}$ & & & \\
Alpha-1-antitrypsin & $49 / 5.0-5.4$ & $0.2(0.0-0.3)$ & $0.7(0.3-1.0)^{*}$ \\
Transthyretin & $14 / 5.5$ & $0.0(0.0-<0.1)$ & $0.1(<0.1-0.2)^{*}$ \\
\hline
\end{tabular}


Figure 1

\section{ApoA-IL202P}
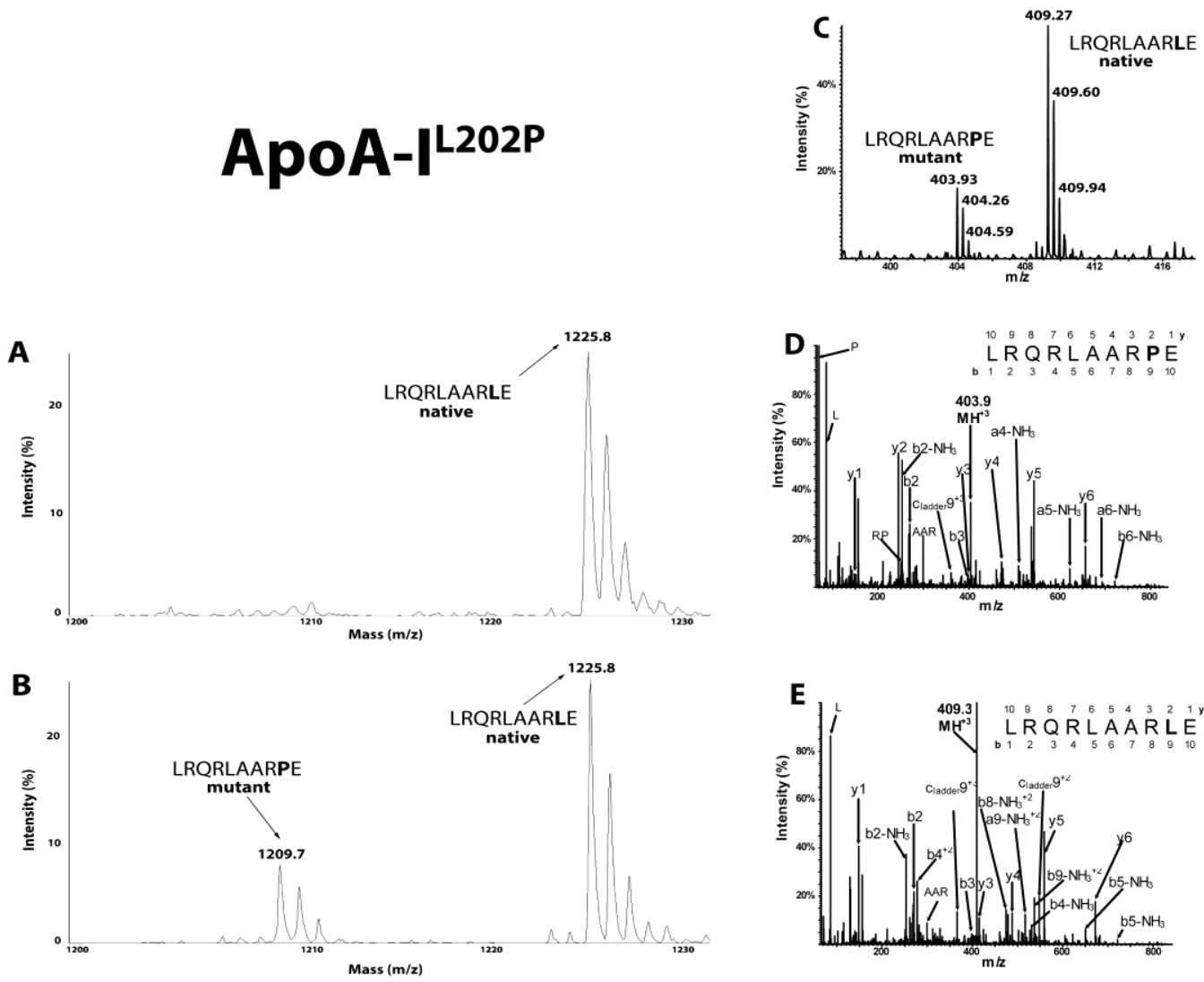
Figure 2

\section{kDa}

35 WT

25

35 Het

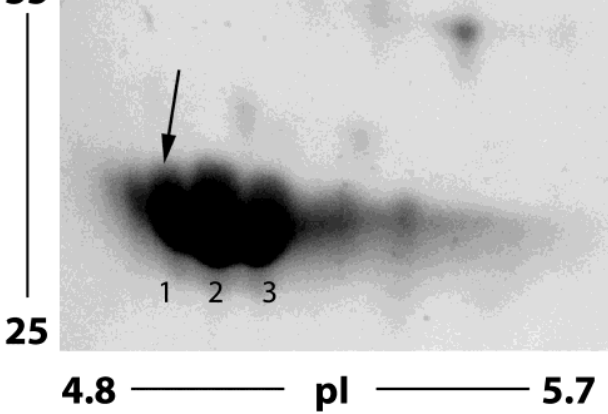


Figure 3

\section{ApoA-I ${ }^{\mathrm{K} 131 \mathrm{del}}$}

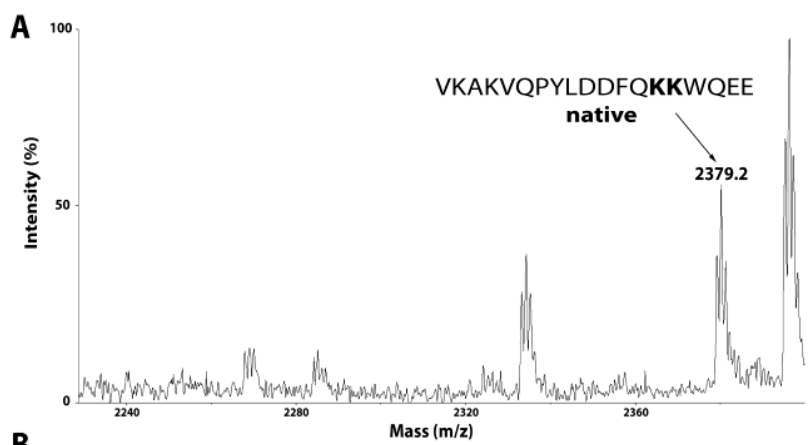

B 100 VKAKVQPYLDDFQKWQEE

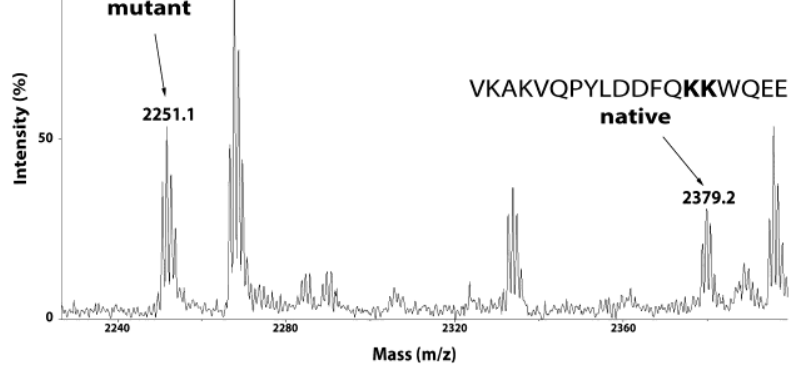

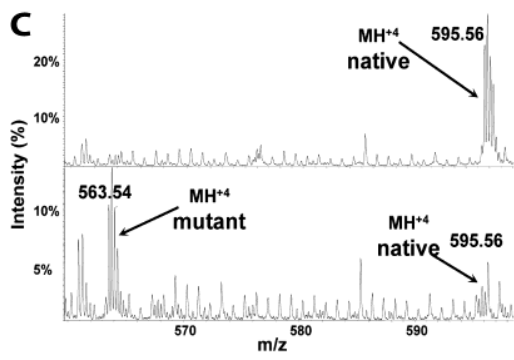

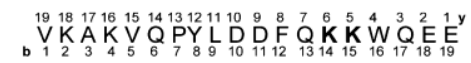
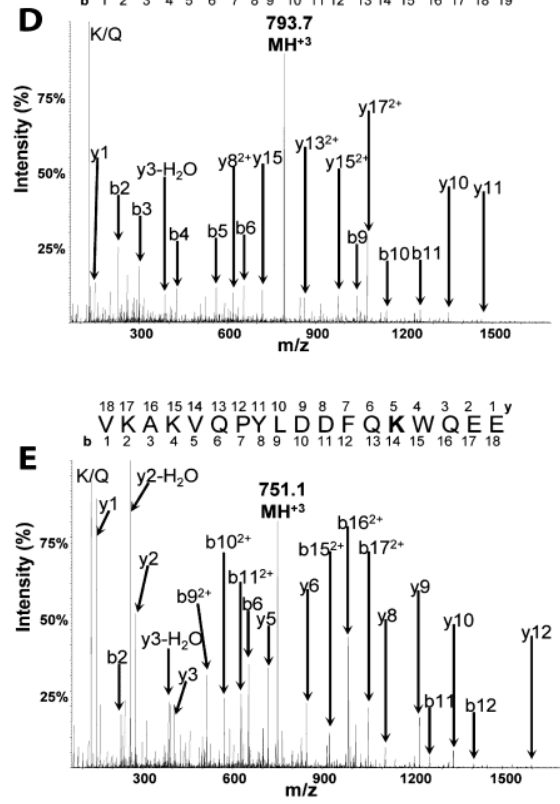
Supplemental Table 1 - Peak area determinations and normalized mutant and wild type peptide proportions.

Peptide peak areas of wild-type peptides, mutant peptides and a control peptide analyzed by MS. The wild-type and mutant peptide peak areas normalized to the control peptide peak area and the mutant/wild-type peptide ratio determined. Values are mean +/- SEM

\begin{tabular}{|c|c|c|c|c|}
\hline & $\begin{array}{l}\text { apoA-I }{ }^{\mathrm{L} 202 \mathrm{P}} \\
\text { Controls }\end{array}$ & $\begin{array}{c}\text { apoA-I }{ }^{\mathrm{L} 202 \mathrm{P}} \\
\text { Heterozygotes }\end{array}$ & $\begin{array}{l}\text { apoA-I }{ }^{\mathrm{K} 131 \mathrm{del}} \\
\text { Controls }\end{array}$ & $\begin{array}{c}\text { apoA-I }{ }^{\mathrm{K} 131 \mathrm{del}} \\
\text { Heterozygotes }\end{array}$ \\
\hline \multirow{5}{*}{$\begin{array}{c}\text { Control Peptide } \\
(\mathrm{m} / \mathrm{z} \text { 1252.7) } \\
\text { Wild-type Peptide } \\
(\mathrm{m} / \mathrm{z} \text { 1225.8 for L202P, } \\
\mathrm{m} / \mathrm{z} \text {. 2379.2 for } \\
\text { K131Del) } \\
\text { Mutant Peptide } \\
(\mathrm{m} / \mathrm{z} 1209.7 \text { for } \mathrm{L} 202 \mathrm{P}, \\
\mathrm{m} / \mathrm{z} 2251.1 \text { for } \\
\text { K131Del })\end{array}$} & Peak area & Peak area & Peak area & Peak area \\
\hline & $4.4 \mathrm{e}^{5} \pm 1.5 \mathrm{e}^{5}$ & $4.8 \mathrm{e}^{5} \pm 2.2 \mathrm{e}^{5}$ & $1.7 \mathrm{e}^{6} \pm 2.5 \mathrm{e}^{5}$ & $1.5 \mathrm{e}^{6} \pm 4.5 \mathrm{e}^{5}$ \\
\hline & $1.1 \mathrm{e}^{5} \pm 2.6 \mathrm{e}^{4}$ & $1.1 \mathrm{e}^{5} \pm 4.7 \mathrm{e}^{4}$ & $6.5 \mathrm{e}^{4} \pm 3.2 \mathrm{e}^{4}$ & $1.1 \mathrm{e}^{4} \pm 1.5 \mathrm{e}^{3}$ \\
\hline & - & $3.3 \mathrm{e}^{4} \pm 1.4 \mathrm{e}^{4}$ & - & $3.9 \mathrm{e}^{4} \pm 1.3 \mathrm{e}^{4}$ \\
\hline & $\begin{array}{c}\text { Proportion } \\
(\%)\end{array}$ & Proportion (\%) & $\begin{array}{c}\text { Proportion } \\
(\%)\end{array}$ & Proportion (\%) \\
\hline Wild-type/Control & $26.2 \pm 3.3$ & $28.3 \pm 6.8$ & $3.5 \pm 2.3$ & $1.0 \pm 0.3$ \\
\hline \multirow{2}{*}{ Mutant/Control } & - & $9.0 \pm 2.3$ & - & $2.7 \pm 0.3$ \\
\hline & & Peptide ratio & & Peptide ratio \\
\hline Mutant/Wild-type & - & $0.3 \pm 0.0$ & - & $3.4 \pm 1.1$ \\
\hline
\end{tabular}


Supplemental Table 2. Peptide mass fingerprinting results

\begin{tabular}{|l|l|l|l|l|l|}
\hline Number & Protein & $\begin{array}{l}\text { Accession } \\
\text { number }^{\mathrm{a}}\end{array}$ & $\begin{array}{l}\mathrm{Mw} \\
(\mathrm{Da} / \mathrm{pI})^{\mathrm{b}}\end{array}$ & $\begin{array}{l}\text { Number of } \\
\text { peptides }^{\mathrm{c}}\end{array}$ & $\begin{array}{l}\text { Sequence } \\
\text { Coverage } \\
\%\end{array}$ \\
\hline 1 & $\begin{array}{l}\text { Zn- } \alpha-2- \\
\text { glycoprotein }\end{array}$ & P25311 & $48000 / 4.6$ & 5 & 18.8 \\
\hline $2 \mathrm{a}$ & Apo E & P02649 & $34000 / 5.4$ & 2 & 5.4 \\
\hline $2 \mathrm{~b}$ & Apo E & P02649 & $34000 / 5.5$ & 8 & 27.1 \\
\hline $2 \mathrm{c}$ & Apo E & P02649 & $34000 / 5.6$ & 23 & 61.5 \\
\hline $3 \mathrm{a}$ & Apo A-I & P02647 & $25000 / 5.2$ & 15 & 37.0 \\
\hline $3 \mathrm{~b}$ & Apo A-I & P02647 & $25000 / 5.3$ & 24 & 65.9 \\
\hline $3 \mathrm{c}$ & Pro Apo A-I & P02647 & $25000 / 5.5$ & 6 & 20.6 \\
\hline $3 \mathrm{~d}$ & Apo A-I (Het) & P02647 & $25000 / 5.1$ & 8 & 32.2 \\
\hline $3 \mathrm{e}$ & $\begin{array}{l}\text { Apo A-I } \\
\text { (WT+Het) }\end{array}$ & P02647 & $25000 / 5.2$ & 14 & 47.2 \\
\hline $3 \mathrm{f}$ & Apo A-I (WT) & P02647 & $25000 / 5.3$ & 15 & 51.7 \\
\hline $3 \mathrm{~g}$ & $\begin{array}{l}\text { Pro Apo A- } \\
\text { I(Het) }\end{array}$ & P02647 & $25000 / 5.4$ & 13 & 47.6 \\
\hline $3 \mathrm{~h}$ & $\begin{array}{l}\text { Pro Apo A- } \\
\text { I(WT) }\end{array}$ & P02647 & $25000 / 5.5$ & 16 & 58.4 \\
\hline 4 & $\alpha-1-a n t i t r y p s i n$ & P01009 & $49000 / 5.0$ & 30 & 60.5 \\
\hline 5 & Transthyretin & P02766 & $15000 / 5.5$ & 5 & 63.3 \\
\hline
\end{tabular}

Proteins of interest were identified by peptide mass fingerprinting using MALDI

TOF MS. Numbers refer to apo A-I isoforms and proteins significantly $(\mathrm{p} \leq 0.05)$

changed in heterozygous HDL ( $n=3)$ compared to wild-type HDL (n=3) also

illustrated in supplemental Figure 2. Peptide mass values were matched with a mass

accuracy $<50 \mathrm{ppm}$.

a) according to UniProt $\mathrm{KB} 2013$ b) experimental c) resulting from Glu-C or Tryptic cleavage as described previously [16]. 

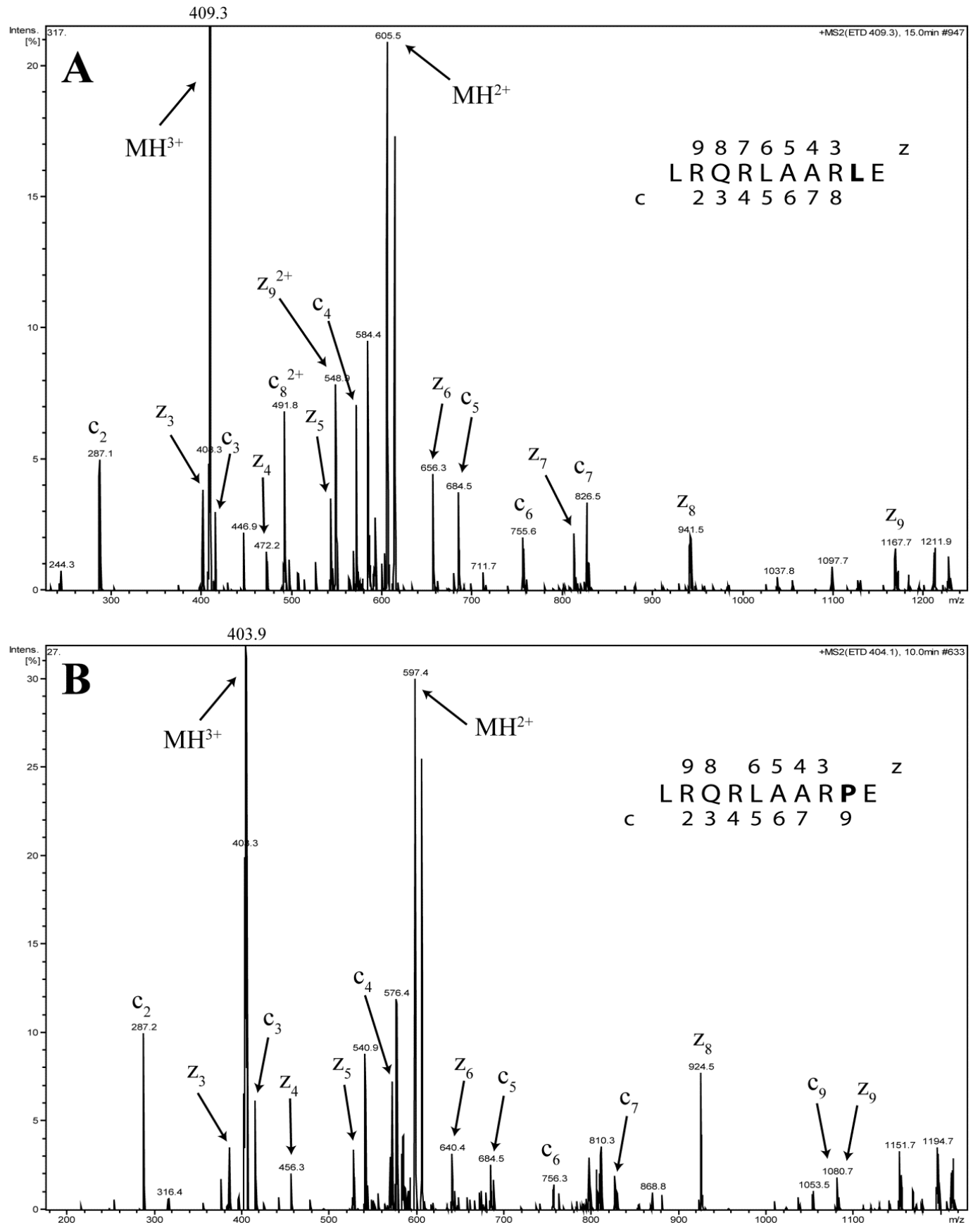

\section{Supplemental Figure 1}

ApoA-I peaks from a L202P heterozygote analyzed by nLC-MS/MS with electrontransfer dissociation. A: fragmentation spectra of $\mathrm{m} / \mathrm{z} 409.3\left(\mathrm{MH}^{3+}\right)$ corresponding to wild-type peptide, B: fragmentation spectra of $\mathrm{m} / \mathrm{z} 403.9\left(\mathrm{MH}^{3+}\right)$ corresponding to the mutated peptide. A proline instead of a leucine was identified in the $\mathrm{c} 9$ ion in the $\mathrm{m} / \mathrm{z}$ $403.9^{3+}$ peptide. 
A

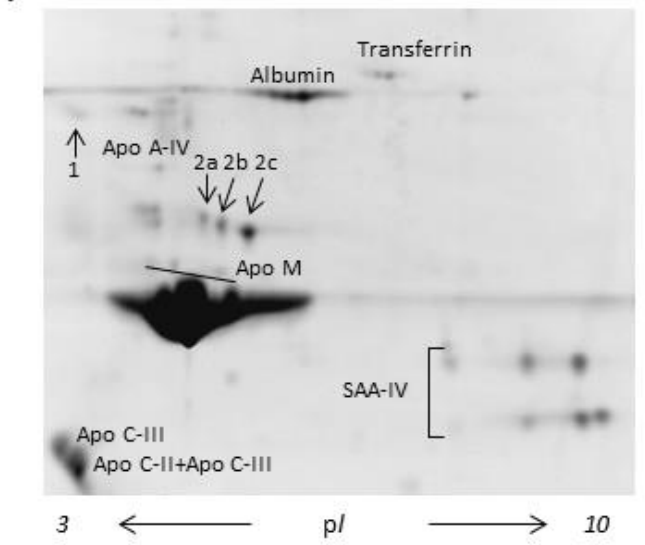

C

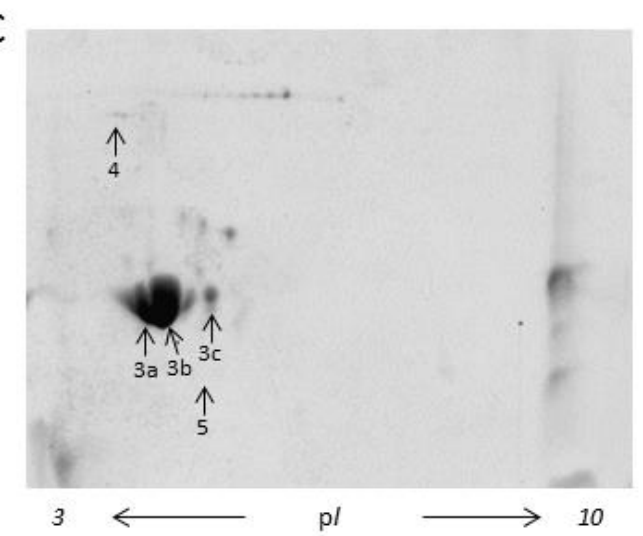

B

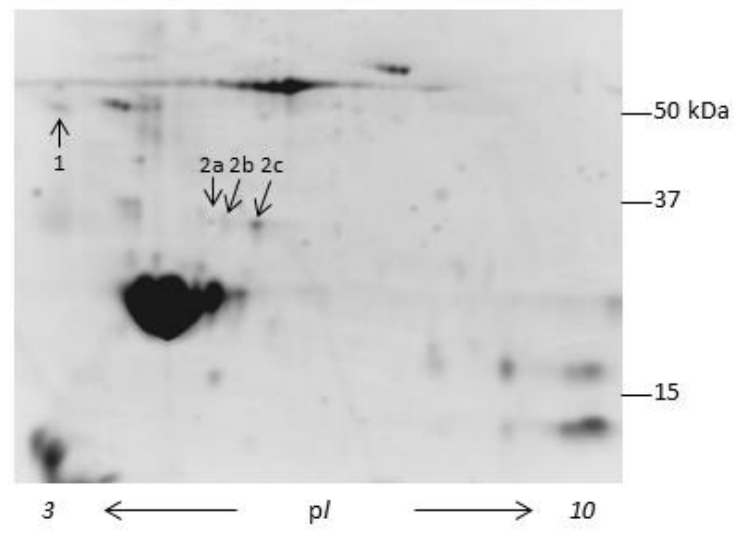

D

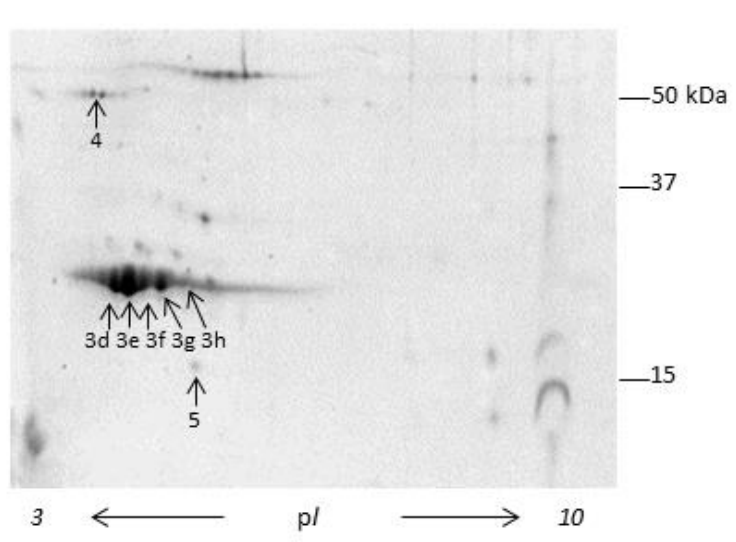

\section{Supplemental Figure 2}

2-DE images of HDL from an apoA-I L202P wild-type control (A), apoA-I L202P heterozygote (B), apoA-I K131del wild-type control (C) and an apoA-I K131del heterozygote (D). 300ug HDL proteins were focused on 3-10NL IPGs and separated on $14 \%$ homogenous gels. Proteins were visualized by Sypro Ruby staining. After quantification, proteins of interest were identified by peptide mass fingerprinting using MALDI TOF MS and search details are presented in supplemental Table 2. Numbers indicate, besides apo A-I isoforms (3a-h), HDL proteins significantly changed in heterozygotes compared to controls; zinc- $\alpha$-2-glycoprotein (1), apo E (2ac), $\alpha$-1-antitrypsin (4) and transthyretin (5). Other proteins indicated according to previous results [16]. 